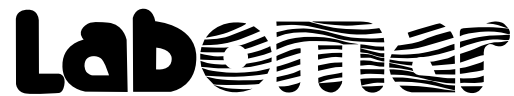

\section{PLANOS TÉCNICOS DE MAQUETES DE REDES DE ARRASTOS COM PORTAS PARA O ENSINO DA TECNOLOGIA PESQUEIRA}

\author{
Technical plans of otter trawls scale models for \\ teaching fishing technology
}

\author{
Natálya de Souza Bernardelli; Eduardo Guilherme Gentil de Farias² \\ ${ }^{1}$ Graduanda em Engenharia de Pesca, Grupo de Tecnologia e Ciência Pesqueira (TECPESCA), \\ Universidade do Estado de Santa Catarina (UDESC) \\ 2 Professor Adjunto II, Grupo de Tecnologia e Ciência Pesqueira (TECPESCA), \\ Universidade do Estado de Santa Catarina (UDESC). e-mail: eduardo.gentil@udesc.br
}

\begin{abstract}
RESUMO
Maquetes físicas podem auxiliar o ensino acerca da confecção e dimensionamento dos petrechos de pesca, possibilitando levantar questões, comunicar ideias e suscitar hipóteses sobre o comportamento destes dispositivos no ambiente aquático, fornecendo um viés alternativo às metodologias tradicionais de ensino da tecnologia pesqueira que, muitas vezes incitam a falta de estímulo por parte dos discentes. Deste modo, o presente trabalho oferta uma perspectiva ainda inédita para o ensino da tecnologia pesqueira no país: a proposta de planos técnicos destinados à confecção de miniaturas de redes de arrasto com portas. Assim, foram dimensionados quatro planos técnicos miniaturizados da modalidade arrasto, contemplando uma ampla diversidade de planos construtivos para esta modalidade de captura. Os planos técnicos sugeridos permitem a obtenção de maquetes com o mesmo formato geométrico dos planos tradicionais. As frações de corte calculadas consideraram o uso de panagens de poliamida multifilamento com abertura de malha $25 \mathrm{~mm}$. Esperamos que a presente proposta possa fomentar novas metodologias didáticas dedicadas ao ensino de projetos de pesca a partir de um processo reflexivo, promovendo a qualificação de recursos humanos capazes de gerar alternativas tecnológicas viáveis para o setor pesqueiro.
\end{abstract}

Palavras-chave: Tecnologia pesqueira, planos técnicos e recursos didáticos.

Recebido em: 22/2/2017

Aprovado em: 14/7/2017

Publicado online em: 20/1/2018 


\section{ABSTRACT}

Physical models can help to teach over the manufacture and design of fishing gears, inciting questions, communicating ideas, and suggesting hypotheses about the behavior patterns of fishing gears in the aquatic environment. In this context, these models provide an alternative tool to the traditional educational approach in fishing technology. So, the present work offer an innovative perspective for fishing technology in Brazil: we proposed four technical plans for the manufacture of miniature otter trawling miniatures, which were designed to cover the construction diversity of construction plans for this fishing method. The suggested technical plans allow to obtain prototype models with the same geometric shape of traditional net plans. The technical plans considered the use of polyamide multifilament with $25 \mathrm{~mm}$ of mesh opening in all models. We hope that this proposal can promote new didactic resources dedicated to fishing gears projects, encouraging the students to a reflective process, enabling the qualification of human resources capable to generate viable technological alternatives for the fishing activity.

Key words: Fishing technology, technical plans and didactic resources.

\section{INTRODUÇÃO}

O dimensionamento das artes de pesca podem ser conduzidos sob diferentes escalas, dependendo do esforço de captura a ser empregado (Sainsbury, 1996). Fazendo um prospecto acerca da transmissão do conhecimento da atividade pesqueira, é razoável afirmarmos que as noções de engenharia empregadas para a obtenção dos petrechos é, historicamente repassada a partir de uma abordagem empírica para diferentes gerações de um mesmo grupo familiar ou comunidade tradicional (Cochrane \& Garcia, 2009).

No entanto, a partir do início do século XX, com o advento da Tecnologia Pesqueira, o conhecimento acerca das artes e métodos de captura pôde ser acessado pela comunidade acadêmica, tendo esta premissa, fomentado à quantificação das técnicas usualmente empregadas pelas comunidades pesqueiras tradicionais (Fridman, 1987). Adicionalmente, a ascensão da atividade pesqueira para patamares industriais, permitiu a mecanização e a automação dos procedimentos de captura, favorecendo a disseminação do conhecimento acerca dos principais petrechos empregados na pesca (Biswas, 2012).

O ensino da confecção e dimensionamento dos petrechos por intermédio da Tecnologia Pesqueira, usualmente é realizado a partir de modelos numéricos ou físicos que favorecem a compreensão acerca do comportamento das redes de pesca no ambiente aquático, possibilitando levantar questões, comunicar idéias e suscitar hipóteses (Okonski \& Martini, 1987; Domingos \& Farias, 2015).

No âmbito acadêmico, os modelos físicos tridimensionais são usualmente denominados como maquetes, sendo estas concebidas a partir de planos técnicos. Entende-se por maquetes como uma representação (completa ou parcial) em escala reduzida de um objeto, sistema ou estrutura, podendo ser estática, se visa analisar o aspecto físico do que está sendo modelado, ou dinâmico, caso o foco seja contemplar o comportamento funcional do que está sendo modelado (Lombardo \& Castro, 1997).

Neste sentido, o dimensionamento de planos técnicos viáveis poderá fomentar a construção de maquetes específicas, estimulando a fixação de assuntos, constituindo-se 
portanto, numa abordagem relativamente inovadora, fornecendo um viés alternativo às metodologias tradicionais de ensino que, muitas vezes incitam a falta de estímulo por parte dos discentes (Freire, 1996).

No caso particular da transmissão de conhecimentos referentes às tecnologias de pesca, o uso das maquetes favorece a apropriação do conhecimento, uma vez que há uma concentração de informações retiradas de formas dimensionais (planos construtivos) a serem apresentadas tridimensionalmente a terceiros, educando os participantes envolvidos no processo de elaboração dos modelos. Entretanto, cabe salientar que na literatura há uma carência quase absoluta de disponibilidade de planos construtivos de maquetes miniaturizadas de petrechos de pesca, sendo este fato, um elemento limitador na transmissão do conhecimento.

Partindo desta premissa, o presente trabalho dimensionou quatro planos construtivos miniaturizados para a confecção de maquetes de redes de arrasto comumente empregadas na atividade pesqueira. A escolha desta modalidade deve-se inicialmente à relevância que este petrecho possui na produção massiva de recursos pesqueiros e, em decorrência dos possíveis impactos ambientais que podem ser desencadeados por esta modalidade de pesca (Broadhurst, 2000). Desta feita, os planos técnicos elaborados neste esforço, poderão suscitar a partir de um processo reflexivo, alternativas tecnológicas que possam mitigar os potenciais efeitos deste petrecho sobre o ambiente aquático.

\section{MATERIAL E MÉTODOS}

Foram dimensionados os planos técnicos para quatro petrechos da modalidade arrasto com portas, tendo estes sido adaptados das plantas originais disponíveis em FAO (1975), FAO (1978), Broadhurst et al. (2012) e Vieira (2015). A escolha buscou contemplar a diversidade de planos construtivos para esta modalidade de captura. Assim, foram concebidos os planos técnicos para arrasto de meia água para peixes, arrasto para peixes de fundo e dois arrastos camaroeiros com padrões de corte distintos.

Os projetos de arrastos eleitos foram: arrasto de meia água para peixes pelágicos, arrasto para peixes demersais e dois planos técnicos distintos para arrastos camaroeiros (mangas e túnel conectados com panagens laterais e mangas e túnel separados sem panagem lateral).

As redes de arrasto foram o foco deste esforço em virtude da relevância econômica desta modalidade de captura para o setor produtivo, complexidade técnica construtiva e potencial impacto destas artes de pesca sobre o ambiente aquático (Andrew et al., 1991; Broadhurst et al., 1996; Domingos et al., 2016). Neste sentido, a obtenção das maquetes tridimensionais poderão servir de suporte para a formação de discentes e técnicos da área de Recursos Pesqueiros e Engenharia de Pesca que possuam interesse específico no âmbito da tecnologia pesqueira.

A obtenção das maquetes seguiu o modelo estático com representação parcial (Simieli, 1991). Os planos técnicos miniaturizados contemplaram a obtenção de maquetes com o mesmo formato geométrico dos planos tradicionais. Contudo, visando reduzir os custos de confecção, o procedimento de miniaturização não reduziu as escalas das aberturas das malhas das panagens, bem como, a espessura dos fios utilizados nos planos construtivos originais.

Todos os planos técnicos foram confeccionados seguindo as normativas previstas em FAO (1990) que adota três padrões básicos de corte de rede: os cortes "N" são aqueles rea- 
lizados no sentido dos nós do pano, em " $\mathrm{T}$ " incidem no sentido dos contra os nós da panagem e, por fim, o corte que incide sobre os lados da malha é indicado pela letra " $\mathrm{B}$ ". Quando precedidos da letra $\mathrm{A}$ (ex: $\mathrm{AB}$ ), indicam que o corte deverá ser realizado ao longo de toda a panagem. Entende-se como pano ou panagem o resultado da conexão pareada de várias malhas no comprimento e altura (com ou sem nós) (FAO, 1990). Maiores detalhes sobre estas definições podem ser encontradas em Domingos \& Farias (2015).

A paridade geométrica entre os planos originais frente aos planos técnicos miniaturizados foi possível graças a adoção da abordagem analítica para frações de corte sugeridas por Fridman (1987) e Domingos \& Farias (2015). As frações de corte calculadas consideraram o uso de panagens de poliamida multifilamento, tendo sido este material utilizado em todas as maquetes, associando às frações analíticas de fração de corte às técnicas construtivas clássicas (recortar, entralhar, perfiar e encabeçar).

\section{RESULTADOS E DISCUSSÕES}

O plano técnico referente ao arrasto para peixes de fundo foi dimensionado considerando a disposição de malhas $25 \mathrm{~mm}$ para todas as panagens (Figura 1). Tal premissa, visou reduzir custos de montagem, priorizando apenas a percepção geométrica da maquete. Esta arte de pesca apresentou sete panos na porção superior e cinco panagens na porção inferior.

O projeto miniaturizado (Figura 1) apresentou 2 frações de corte distintas na porção interna das mangas (1N2B e AB) e cortes em AN na porção externa. Cabe salientar que 3 panagens devem ser concatenadas para a obtenção das mangas superiores, visando assim, simular o incremento da abertura vertical destes petrechos (Okonsky \& Martini, 1987). Após serem submetidas à fração de corte, as panagens superiores apresentaram as seguintes frações: inicialmente 1N2B, em seguida $A B$, na sequência tivemos seis frações 1N2B consecutivas e, por último AN. As panagens inferiores apresentaram frações 3N2B, seguidas por três frações 1N2B no túnel e AN no ensacador.

Visando obter um plano técnico de miniatura de petrecho empregado para a captura de peixes pelágicos, dimensionamos uma rede de arrasto de meia água para este propósito (Figura 2). O projeto considerou um planta com quatro panagens principais (isto é, incluindo 2 panagens laterais). Diferentemente do petrecho disposto na Figura 1, neste dispositivo miniaturizado as malhas da porção das mangas (asas) e boca apresentam um decréscimo contínuo da abertura das malhas empregadas. Assim, foram concebidas carreiras com malhas $8,7,6$ e $5 \mathrm{~cm}$ (entre nós opostos), sendo as maiores aberturas de malhas verificadas nas pontas das mangas e as menores nas carreiras que circundam a boca do referido petrecho, sendo esta abordagem comum à esta modalidade de captura (FAO, 1978).

Salientamos que as panagens superior e inferior do petrecho miniaturizado destinado à captura de peixes pelágicos contou com frações de corte 2N1B no túnel e AN nas panagens do ensacador. Nas panagens laterais, o túnel apresentou as seguintes frações de corte: 2N1B, 9N2B e 2N1B, respectivamente (sentido túnel-ensacador). Por fim, o ensacador apresentou a mesma fração de corte observada para as panagens superior e inferior (AN).

Na sequência, foram dimensionados os planos técnicos que contemplaram as pescarias de camarões marinhos. Neste sentido, foram escolhidos dois projetos distintos. Inicialmente, consideramos um plano técnico que integra as mangas ao túnel e que apresenta panagens laterais. Dispositivos para a captura de camarões com este layout são usuais nos ambientes transicionais da Oceania (Broadhurst et al., 1996), sendo as panagens laterais 


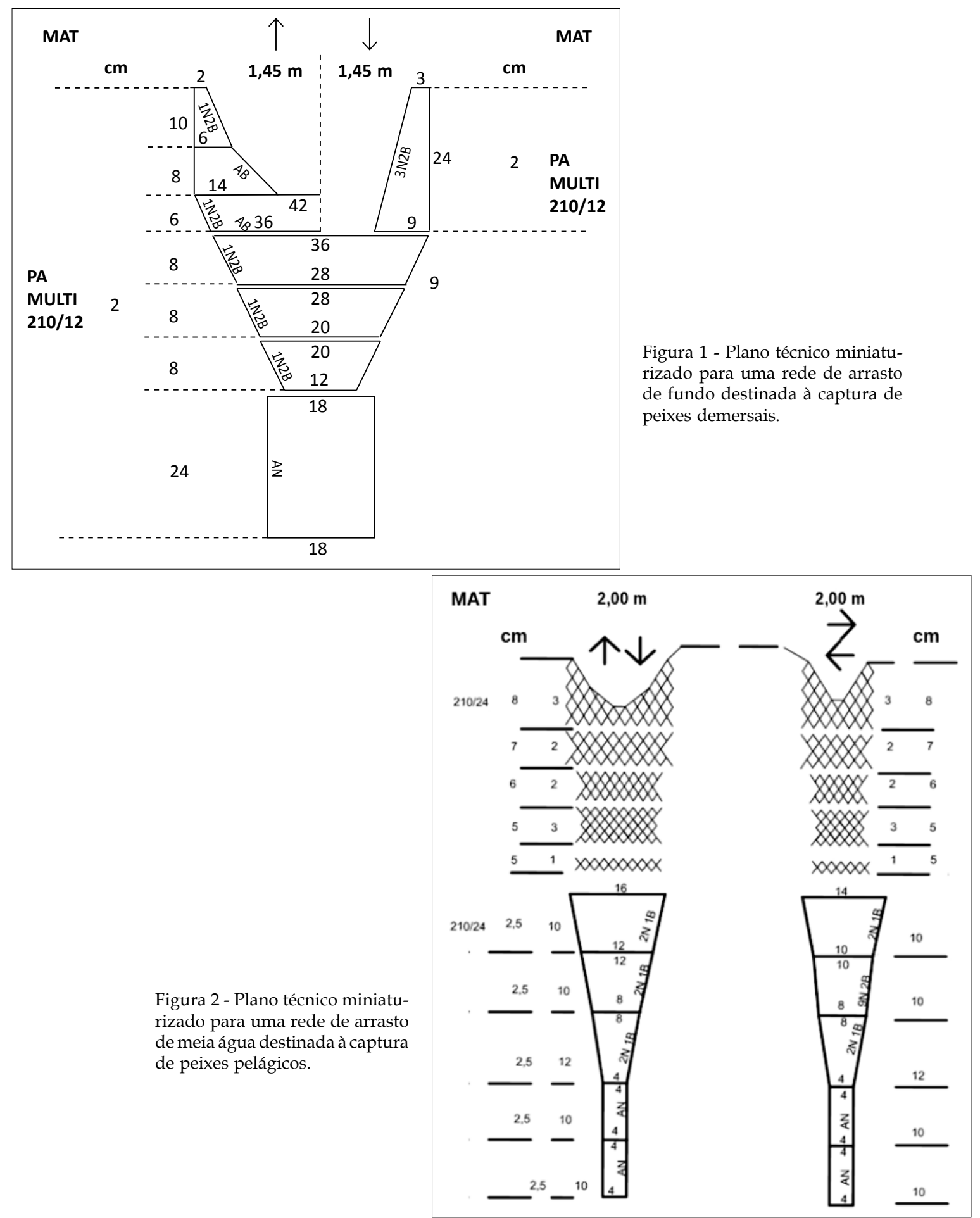

para esta modalidade, responsáveis por limitar a abertura vertical (Domingos et al., 2016). Os petrechos miniaturizados destinados à captura de camarões também apresentaram malhas com $25 \mathrm{~mm}$ (distância entre nós opostos). É pertinente salientar que neste plano técnico (Figura 3), as panagens superiores e inferiores são idênticas. Neste sentido, as panagens laterais seguem a mesma lógica, isto é, são similares. Contudo, diferentemente dos panos das mangas + túnel, estes apresentam formato retangular e frações de corte AN no comprimento e AT na altura (Figura 3). 
Figura 3 - Plano técnico miniaturizado para uma rede de arrasto camaroeira com quatro panagens + integração mangas e túnel.

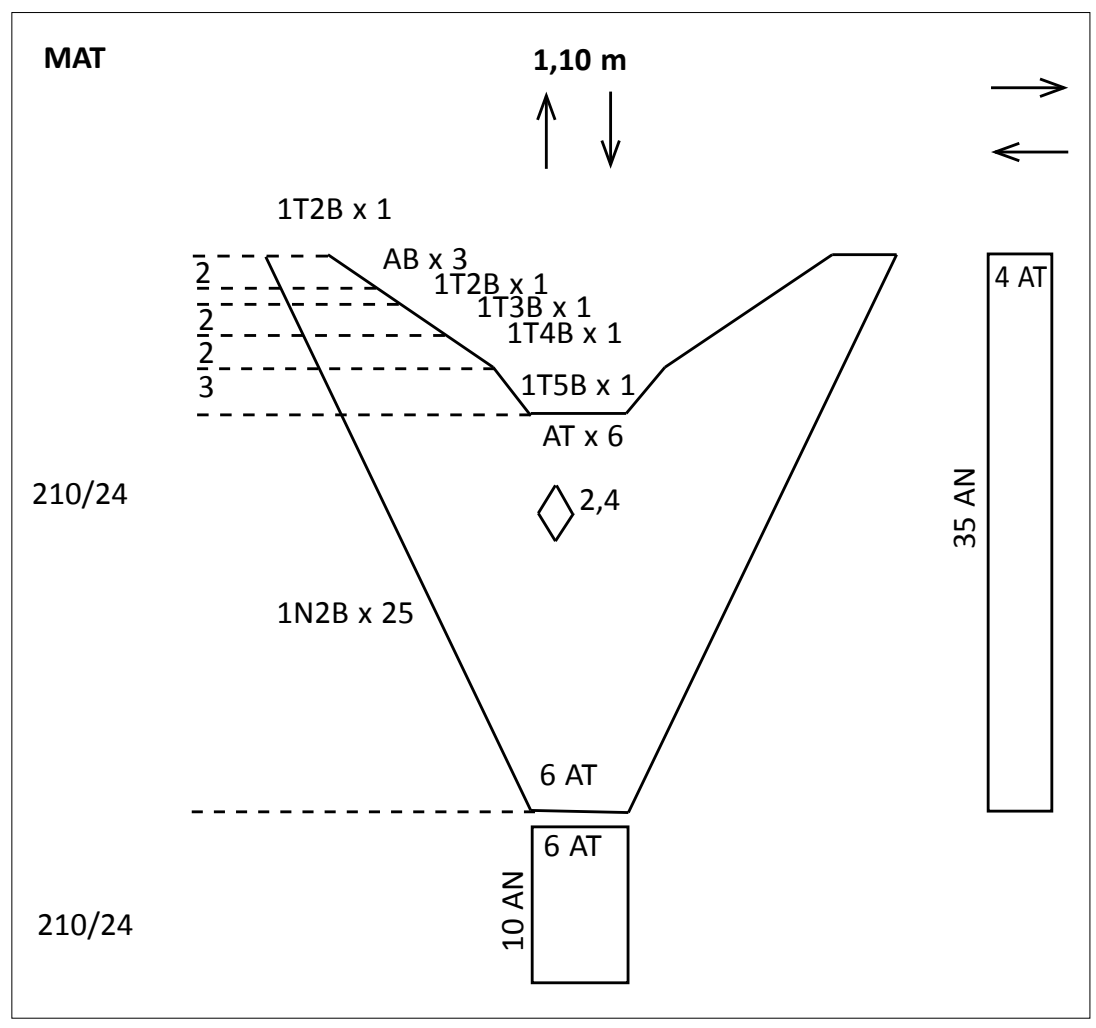

Por fim, foi dimensionada uma planta técnica de um arrasto camaroeiro tipicamente empregado pela frota brasileira (Figura 4). Este petrecho apresenta duas panagens básicas (superior e inferior), com ausência de panagens laterais e emprego de um único pano unindo as mangas e o túnel. O plano técnico contou com duas frações de cortes distintas para as mangas (2B1N na porção interna e 1B2N na porção externa) e uma única para o túnel (2B1N). Por fim, o ensacador contou com cortes AN e AT. É importante ratificar que todos os planos técnicos preservaram as características geométricas encontradas nos petrechos com escala real.

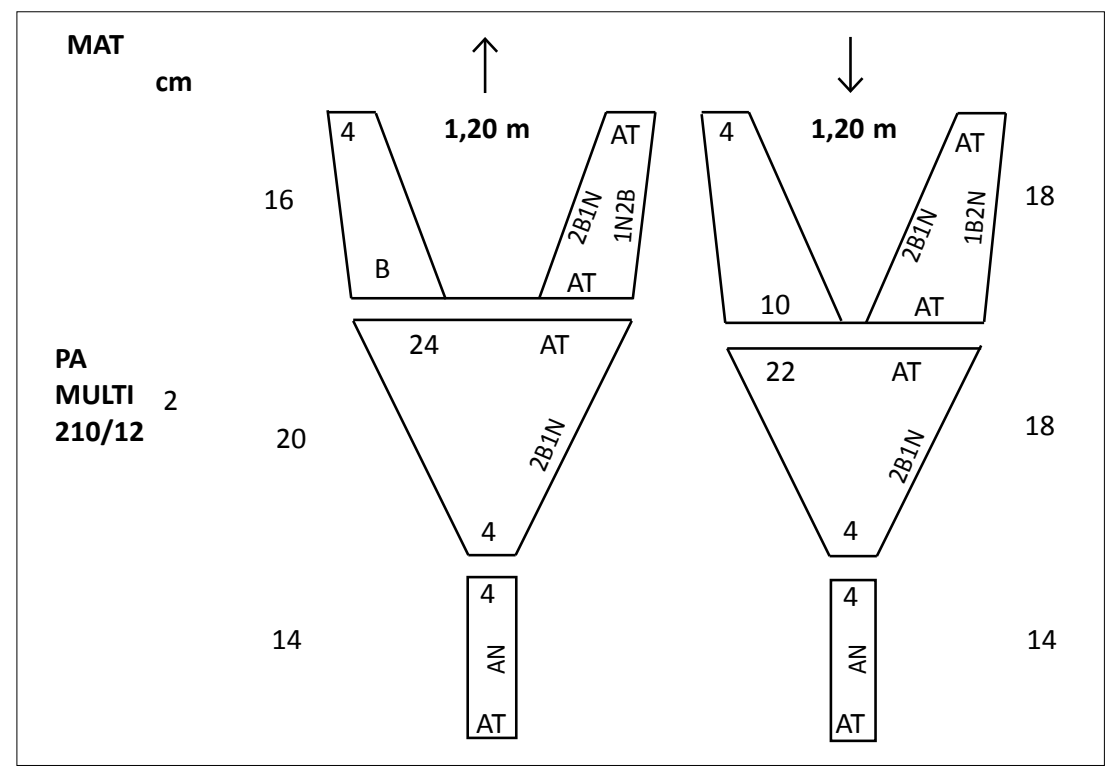

Figura 4: Plano técnico miniaturizado para uma rede de arrasto camaroeira com duas panagens (design típico das frotas comerciais das regiões Sudeste e Sul do Brasil). 


\section{CONCLUSÕES}

O presente esforço sugere a elaboração de maquetes a partir de planos técnicos específicos dimensionados para esta finalidade. Esta abordagem possibilita aos discentes da área de Tecnologia Pesqueira, a utilização de informações concretas de petrechos em escala reduzida, favorecendo a interpretação e o entendimento acerca do comportamento das artes de arrasto com portas em ambientes aquáticos, permitindo os alunos partirem de uma visão bidimensional para uma visão tridimensional dos petrechos representados.

Ao nosso conhecimento, o esforço apresentado neste trabalho constitui-se como uma iniciativa pioneira no âmbito pedagógico da Tecnologia Pesqueira. Adicionalmente, a adoção das maquetes calculadas e dimensionadas neste trabalho, poderá fomentar uma nova abordagem pedagógica que aproxime o conhecimento teórico à realidade do setor produtivo, fomentando inclusive, novas linhas interpretativas consoantes a seletividade, captura e métodos de exploração racional de recursos pesqueiros.

\section{REFERÊNCIAS}

Andrew, N. L.; Graham, K. J.; Kennelly, S. J. \& Broadhurst, M. K. The effects of trawl configuration on the size and composition of catches using benthic prawn trawls off the coast of New South Wales, Australia. ICES J Mar Sci., v. 48, p. 201-209, 1991.

Biswas, K. P. Advances in Fishing Technology. New Delhi: Daya Publishing House. 343 p., 2012. Broadhurst, M. K.; Kennelly, S. J. \& Isaksen, B. Assessments of modified codends that reduce the by-catch of fish in two estuarine prawn-trawl. fisheries in New South Wales, Fish. Res., v. 27, p. 89-111, 1996.

Broadhurst, M. K. Modifications to reduce bycatch in prawn trawls: A review and framework for development. Rev. Fish Biol. Fish., v. 10, p. 27-60, 2000.

Cochrane, K. L. \& Garcia, S. M. A Fishery Manager's Guidebook. 2a ed. Oxford (UK): WileyBlackwell. 536 p., 2009.

Domingos, M. M. \& Farias, E. G. G. Aplicação dos cálculos de fração de corte para redes de pesca. Bol. Inst. Pesca, v. 41, n. 4, p. 1049-1057, 2015.

Domingos, M. M.; Rodrigues-Filho, J. L. \& Farias, E. G. G. Avaliação de uma rede de arrasto de fundo estruturalmente modificada. Bol. Inst. Pesca, v. 42, n. 2., p. 353-368, 2016.

Food and Agriculture Organizatin (FAO). Catalogue of small scale fishing gear. Oxford (UK): FAO Fishing News Books Ltd. 191p., 1975,

Food and Agriculture Organizatin (FAO). Catalogue of fishing gear designs. Oxford (UK): FAO Fishing News Books Ltd. 160p., 1978.

Food and Agriculture Organizatin (FAO). Fisherman's Workbook. Oxford (UK): FAO Fishing News Books Ltd. 155p., 1990.

Freire, P. Pedagogia da Autonomia: saberes necessários à prática educativa. 36a ed. São Paulo: Paz e Terra. 144 p., 1996.

Fridman, A. Calculations for Fishing Gear Designs. Oxford (UK): FAO Fishing News Books Ltd. 185p., 1987. 
Lombardo, M. A. \& Castro, J. F. M. O uso de maquete como recurso didático. Rev. Geog. Ens., v. 6, n. 1, p. 81-83, 1997.

Okonski, S. \& Martini, L. W. 1987 Artes y métodos de pesca: Materiales didacticos para la capacitacion técnica. Argentina: Hemisfério sur. 339p.

Sainsbury, J. C. Commercial fishing methods: An introduction to vessels and gears. $3^{\text {a }}$ ed. United Kingdon: Fishing News Books Ltd. 359 p., 1996.

Simieli, M.E.R. O uso de plantas e mapas na escola de $1^{0}$ grau: ênfase para as séries iniciais. In: Projeto Ensino da cidade de São Paulo. São Paulo: Assoc. Geógr. Bras., 1991, p. 24-40

Vieira, W. J. Avaliação do uso de tirantes e do dispositivo kite escape em redes de arrasto camaroeiras, Santa Catarina, Brasil. Santa Catarina. Trabalho de Conclusão de Curso. Bacharelado em Engenharia de Pesca. Universidade do Estado de Santa Catarina, UDESC 60 f., Santa Catarina, 2015. 